\title{
CHARACTERIZATION AND SUSCEPTIBILITY PATTERN OF CANDIDA ISOLATES FROM HIV - SEROPOSITIVE PATIENTS IN A TERTIARY CARE HOSPITAL
}

Apurba Sankar Sastry, Sandhya Bhat K, Anand Sankar Sastry, Kumudavathi M S,

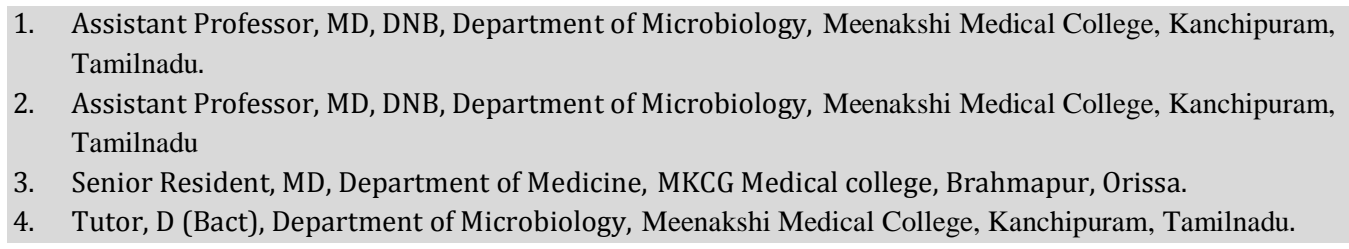

\section{CORRESPONDING AUTHOR}

Dr. Apurba Sankar Sastry, Assistant Professor,

Department of Microbiology,

Meenakshi medical college and Research Institute,

Kanchipuram, Near Chennai, India

E-mail: drapurbasastry@gmail.com,

Ph: 0091444327314.

ABSTRACT: BACKGROUND: Difference in expression of virulence factors and in antifungal susceptibility among different Candida species has raised the need for species-level identification. Opportunistic infection is usually caused by Candida albicans, however, over the last decade reports of non-albicans Candida causing this infection is increasing. AIM: The present study was undertaken to assess the role of various Candida species as opportunistic pathogens in Human Immunodeficiency Virus (HIV) seropositive patients and testing their antifungal susceptibility pattern. METHODS AND MATERIAL: 100 clinical samples were obtained from HIV seropositive patients and 50 from HIV seronegative patients and were speciated by the conventional methods and also by HiCrome Candida Differential Agar (CHROM agar). Susceptibility testing of the isolates for Fluconazole and Itraconazole was performed by Macro broth dilution technique. RESULTS: Candida albicans was the commonest species isolated followed by C.dubliniensis, C.parapsilosis, C.tropicalis, C.krusei and C.glabrata. Antifungal susceptibility testing from HIV seronegative patients showed all Candida albicans and Candida tropicalis isolates were sensitive to both Fluconazole and Itraconazole. Candida krusei ( 1 isolate), Candida glabrata ( 1 isolate), Candida dubliniensis ( 3 isolate) showed resistance to Fluconazole and Candida parapsilosis (1 isolate) showed dose-dependent susceptibility with MIC $=16 \mu \mathrm{g} / \mathrm{ml}$. Itraconazole resistance was observed for Candida krusei $(1$ isolate), Candida glabrata ( 1 isolate), with an MIC range of $\geq 1 \mathrm{mg} / \mathrm{L}$. All other isolates, even which were resistant to Fluconazole were sensitive to Itraconazole. All Candida isolates from HIV seronegative patients were sensitive to both Fluconazole and Itraconazole. CONCLUSION: Increasing trend of Non albicans Candida species causing various invasive infections and their prevalence in a particular geographical area warrant the need of speciation of Candida species. The long-term use of azoles as prophylaxis for Invasive candidiasis can result in the selection of Candida isolates that are more resistant to azole therapy. The non albicans Candida species like C.glabrata and C. krusei are more resistant to Fluconazole and Itraconazole compared to C. albicans. Hence the antifungal susceptibility pattern plays an important role for successful treatment of Candidiasis. The use of Macrobroth dilution method using BHI broth technique will help in detecting the antifungal susceptibility pattern in a less equipped laboratory. 
KEYWORDS: Antifungal susceptibility, Human Immunodeficiency Virus (HIV), ChromAgar

\section{Full text: Isolation and antifungal susceptibility testing of Candida isolates from various clinical samples in Human Immunodeficiency Virus (HIV) Seropositive Patients and HIV Seronegative Patients.}

INTRODUCTION: Candida species are well known as a member of the normal flora as well as opportunistic pathogens. Over the past few decades, the number of opportunistic infections caused by Candida spp has been increased tremendously. The indiscriminate long-term use of antibiotics, indwelling intravascular catheters, immunosuppresives, cytotoxic therapies, immune defects and more recently AIDS patients are among the significant risk factors associated with Candidiasis 1, 2, 3. In the last decade however, the incidence of infections due to Candida albicans have decreased and been replaced by non-albicans Candida spp like Candida tropicalis, Candida parapsilosis, Candida glabrata and Candida krusei with more reduced susceptibility to antifungal agents ${ }^{4,5}$.

The potential clinical importance of species level identification important as various Candida isolates differ in the expression of putative virulence factors and antifungal susceptibility 2, 6. The increased isolation rates of non-albicans Candida species and a gradual shift in the antifungal susceptibility profile to azole antifungal agents, especially in immunocompromised patients, have warranted the need to diagnose the pathogen quickly as well as monitor the laboratory data for possible emergence of resistance 7,8,9.

The available methods for antifungal susceptibility testing are Micro and Macro Broth Dilution method, Agar Dilution method, Semisolid Agar Dilution method and E-test. Increasing reports of non-albicans Candida species with drug resistance causing infections and increasing number of HIV seropositive patients motivated us to undertake the study to differentiate various Candida spp to find out the most prevalent species and to perform antifungal susceptibility testing to know the difference in the susceptibility pattern between HIV seropositive and seronegative patients by Macrobroth dilution method using Brain Heart Infusion broth (BHI) for Fluconazole and Itraconazole.

MATERIAL AND METHODS: A total of 150 clinical samples (oral swab, blood, vaginal swab, urine) were obtained from HIV seropositive (100) and HIV seronegative (50) patients (clinically suspected cases of candidiasis) attending to outpatient department or admitted in the hospital at Meenakshi medical college, Kanchipuram from May 2009 to November 2010, after taking informed written consent. None of our patients were on antifungal drugs during sample collection. With universal precautions, samples were collected and transported to the laboratory without delay and were processed.

Samples were inoculated on Sabouraud's dextrose agar (SDA). Additionally, samples were also inoculated on HiCrome Candida Differential Agar (CHROM agar) (Hi Media Pvt Ltd, Mumbai) for early presumptive identification of various Candida species, based on colored colony morphology (table-1). SDA slants and CHROM agar plates were incubated at $37^{\circ} \mathrm{C}$ for 48 $\mathrm{hr}$. Isolates were further characterized by performing the following conventional techniquesGerm tube production, chlamydospore formation on corn meal agar, sugar assimilation profile and differential growth on SDA at $37{ }^{\circ} \mathrm{C}$ and $42^{\circ} \mathrm{C}$ 8,10-11. Gram staining of all the isolates was done from SDA and microscopic morphology was noted (figure-1).

Journal of Evolution of Medical and Dental Sciences/Volume1/ Issue4/October - 2012 Page 590 
Antifungal susceptibility testing of all the isolates for Fluconazole and Itraconazole was performed by Macro broth dilution technique according Clinical and Laboratory Standards Institute (CLSI) guidelines. For each isolate Minimum Inhibitory Concentration (MIC) for Fluconazole was calculated as the lowest concentration at which $80 \%$ inhibition of growth occurred relative to that of drug free control, and MIC for Itraconazole was calculated as the lowest concentration at which $50 \%$ reduction of turbidity was produced, as compared with that For the drug-free control 12-13.

ANALYSIS OF RESULTS: CLSI breakpoints were used for Fluconazole and Itraconazole. Isolates were considered susceptible for Fluconazole if their MIC was $\leq 8 \mu \mathrm{g} / \mathrm{mL}$, susceptible-dose dependent (S-DD) if their MIC was $16-32 \mu \mathrm{g} / \mathrm{mL}$, and resistant if their MIC was $\geq 64 \mu \mathrm{g} / \mathrm{mL}$ and for Itraconazole susceptible if their MIC was $\leq 0.125$, susceptible-dose dependent (S-DD) if their MIC was $>0.125$ to $<1$ and resistant if their MIC was $\geq 1 \mathrm{mg} / \mathrm{L}$ determined after $48 \mathrm{~h}$ of incubation ${ }^{12-15}$.

RESULTS: Out of 100 HIV seropositive patients, Candida species were isolated from 48 samples while 52 samples showed no growth. Out of 48 samples with growth, one Candida species was isolated in 44 samples and mixture of 2 Candida species in 4 samples. (Table-2)Out of 52 isolates, 40 were isolated from oral thrush, 6 from urine, 3 from blood, and 3 from vaginal swab.

Candida albicans was the commonest species isolated (28 strains) followed by 13 strains of C. dubliniensis, 5 strains of C.parapsilosis, 3 strains of C.tropicalis, 2 strains of C.krusei and 1 strain of C.glabrata were identified (fig-2).

Out of 50 HIV seronegative patients, Candida species were isolated from 24 samples while 26 samples showed no growth. Out of 24 samples with growth, one Candida species was isolated in 20 samples and mixture of 2 Candida species in 4 samples. Out of 28 isolates, 24 were isolated from oral thrush, from urine and 4 from vaginal swab. (Table-2)

Candida albicans was the commonest species isolated (18 strains) followed by 6 strains of C. dubliniensis, 3 strains of C.parapsilosis, 1 strain of C.tropicalis were identified.

Out of 40 Candida isolates from oral thrush, 24 isolates were C.albicans, 13 isolates were C.dublinienis, 2 isolates were C.tropicalis and 1 isolate was C.parapsilosis.

Antifungal susceptibility testing of Candida isolates from HIV seropositive patients showed all Candida albicans and Candida tropicalis isolates were sensitive to both Fluconazole and Itraconazole (100\%) and Candida krusei (1 isolate), Candida glabrata (1 isolate), Candida dubliniensis (3 isolates) showed resistance to Fluconazole with MICs of $64 \mu \mathrm{g} / \mathrm{ml}$ at $48 \mathrm{hr}$, and Candida parapsilosis ( 1 isolate) showed dose-dependent susceptibility with MIC = 16 $\mu \mathrm{g} / \mathrm{ml}($ figure3).

Itraconazole resistance was observed for Candida krusei (1 isolate), Candida glabrata (1 isolate), with an MIC range of $\geq 1 \mathrm{mg} / \mathrm{L}$. All other isolates, even which were resistant to Fluconazole were sensitive to Itraconazole. Out of 13 isolates of Candida dubliniensis, three showed resistance (23\%) to Fluconazole but all were sensitive $(100 \%)$ to Itraconazole (figure4). All these resistant strains were isolated from oral thrush. One out of 5 isolates of Candida parapsilosis showed dose dependant susceptibility to Fluconazole, which was also isolated from oral thrush.

Antifungal susceptibility testing of Candida isolates from HIV seronegative patients showed all Candida albicans and non albicans Candida isolates were sensitive to both Fluconazole and Itraconazole (100\%) and no antifungal drug resistance observed. 
DISCUSSION: C.albicans and non-albicans Candida are opportunistic yeasts, causing severe disease in immunocompromised individuals, like in AIDS patients ${ }^{17}$. Oropharyngeal Candidiasis is one of the most common opportunistic infection in patients with AIDS. Infact oropharyngeal Candidiasis is known to be an AIDS indicator disease ${ }^{4}$. In the present study out of 52 Candida isolates, C.albicans (53.8\%) was the predominant isolate followed by Candida dubliniensis (25\%), which was similar to the study done by Lopez-Dupla et al in 1992 who reported $44.4 \%$ isolation of C.albicans and Wabale et al in 2010 (73.3\%) from HIV seropositive patients ${ }^{4,18}$.

In the present study out of 52 Candida isolates from HIV seropositive patients, 40 Candida species were isolated from oral thrush. Out of 40 Candida isolates from oral thrush, 24 isolates were C.albicans, 13 isolates were C.dublinienis, 2 isolates were C.parapsilosis and 1 isolate was C.tropicalis.

Non albicans Candida as the causative agent of oral thrush have been reported from various places ranges from $6.55 \%-70 \%$. In our study the isolation rate of non albicans Candida species from HIV seropositive patients from oral thrush was 16/40 i.e.40\%. Majority of non albicans Candida isolated were C.dubliniensis (13/16). Sullivan et al reported 64 C.dubliniensis from 55 Irish and Australian HIV seropositive patients ${ }^{19}$.

The increasing emergence of "non-albicans Candida" thus seems to be associated with HIV pandemic. Since C. dubliniensis closely resembles C.albicans phenotypically it is possible that it is being missed in most of laboratories were only germ tube is solely used of the identification of C.albicans. Emergence of C. dubliniensis infection in HIV seropositive patients is a matter of concern due to the emergence of resistance to commonly used azole antifungals ${ }^{4}$.

In our study, all the resistant Candida spp. was isolated from oral thrush. In contrast to our study, resistance was observed for Candida spp. isolated from blood in study by Canan et al in 201015. This could be because of low number of blood isolates in our study.

The use of automated systems such as API20 is unaffordable due to its high cost. Conventional sugar assimilation testing methods can be used for identification, but it takes long time and procedure is cumbersome. Molecular based techniques such as PCR and DNA fingerprinting are very useful in speciation but are not readily available in routine microbiology laboratories ${ }^{20}$.

The colonies of the Candida species can be distinguished following primary isolation from clinical specimens by using CHROM agar medium, which is very much cost effective. CHROM agar shows sufficient sensitivity to grow the most important yeasts. It can serve as primary isolation and differentiation medium for clinical specimens likely to contain yeasts. A major advantage is the ability to detection of mixed cultures of yeast in clinical specimens. Hence CHROM agar Candida would be a useful, cost effective tool for rapid identification of various Candida species directly from clinical samples 2, 5, 9, 21.

In the present study, CLSI reference Macrobroth dilution method (M27-A3) was used to determine the susceptibility of 52 Candida isolates to Fluconazole and Itraconazole. The ability to determine MIC results within 24 hour is potentially advantageous for early clinical application of antifungal susceptibility results. The present study also performed 24 hour and 48 assessments, and only a few isolates required 48 hour of incubation for optimal growth 13 . Antifungal susceptibility testing revealed resistance to Fluconazole among C. dubliniensis isolates, where as all C.albicans isolates were sensitive. 3 (23\%) isolates of C. dubliniensis were resistant to Fluconazole with MIC range of $64 \mu \mathrm{g} / \mathrm{ml}$, which matches with observation made by Chunchanur SK et al $(22.7 \%)^{8}$. However, isolates of C. dubliniensis resistant to Fluconazole may still be sensitive to other azole compounds such as Itraconazole and Voriconazole ${ }^{22}$, which 
matches with our study results. Recently there has been an increased incidence of treatment failures in Candidiasis patients receiving prolonged Fluconazole ${ }^{23}$. Replacement of C. albicans by C. dubliniensis is known to occur in patients treated with Fluconazole. The antifungal pressure exerted by this drug influences the oral microbial ecology in these patients, as species that are better able to adapt to antifungal pressure persist over those that are suppressed by the treatment 8,24 .

The second most common non-albicans Candida species overall isolated from all the samples in our study was C. parapsilosis 5/24(20.8\%) which was similar to other reports documented elsewhere 18,26 .

The morbidity and risk associated with Candidiasis, along with increased incidence of treatment refractory Candidiasis as well as the high incidence of AIDS, makes it important that species identification of Candida isolates should be done along with antifungal susceptibility testing in most of the tertiary care laboratories ${ }^{25}$. The use of Macrobroth dilution method using BHI broth, for antifungal susceptibility testing will help in detecting the antifungal susceptibility pattern in a r labource constrained

CONCLUSION: Though Candida albicans continues to the common \& important pathogen among the Candida spp, there is a drastic increase in the incidence of other species like Candida krusei, Candida tropicalis, Candida glabrata and Candida parapsilosis. Hence identification of Candida to the species level has become compulsory to assist the selection of appropriate antifungal agents in treatment of invasive candidiasis because most of the Non albicans Candida especially Candida glabrata and Candida krusei exhibit reduced Fluconazole susceptibility. The long-term use of azoles in the prophylaxis of systemic mycoses can result in the selection of Candida isolates that are more resistant to azole therapy. Hence rapid species identification and susceptibility testing is of great need for treating physician. Proper identification of Candida species, their prevalence in a particular geographical area and studying their antifungal susceptibility pattern is important for successful treatment of Candidiasis. The use of Macrobroth dilution method using BHI broth technique will help in detecting the antifungal susceptibility pattern in a less equipped laboratory. Although the number of Candida species and antifungal agents used in the present study were limited, the results provide a preliminary idea about the isolates susceptibility patterns. Even though our hospital is a tertiary care centre, none of the Candida albicans isolates showed the resistance pattern to Fluconazole and Itraconazole. Hence clinicians can use these two azole antifungals to treat the patients with candidiasis in our hospital setting. However, our study has few limitations, as we have obtained very low no. of isolates from blood, urine and vaginal swab compared to oral swabs; we were not able to draw any clear significance from theses samples.

\begin{tabular}{|l|l|l|l|}
\hline $\begin{array}{l}\text { Candida } \\
\text { species }\end{array}$ & Colony colour & $\begin{array}{l}\text { No. of isolates from } \\
\text { HIV seropositive } \\
\text { patients (n-52) }\end{array}$ & $\begin{array}{l}\text { No. of isolates from HIV } \\
\text { seronegative patients (n-28) }\end{array}$ \\
\hline Candida albicans & Light-green & 28 & 24 \\
\hline $\begin{array}{l}\text { Candida } \\
\text { dubliniensis }\end{array}$ & Dark-green & 13 & - \\
\hline $\begin{array}{l}\text { Candida } \\
\text { parapsilosis }\end{array}$ & $\begin{array}{l}\text { Cream to pale } \\
\text { pink }\end{array}$ & 5 & 3 \\
\hline Candida & Blue with pink & 3 & 1 \\
\hline
\end{tabular}

Journal of Evolution of Medical and Dental Sciences/Volume1/ Issue4/October - 2012 Page 593 
ORIGINAL ARTICLE

\begin{tabular}{|l|l|l|l|}
\hline tropicalis & halo & & \\
\hline Candida krusei & Pink & 1 & - \\
\hline Candida glabrata & Pink & 1 & - \\
\hline
\end{tabular}

Table-1-Guideline for speciation of various Candida isolates CHROM agar medium ${ }^{16}$ and the results of various Candida species isolated

\begin{tabular}{|l|l|l|l|l|l|l|}
\hline $\begin{array}{l}\text { HIV } \\
\text { serological } \\
\text { status }\end{array}$ & $\begin{array}{l}\text { Total } \\
\text { number of } \\
\text { patients }\end{array}$ & $\begin{array}{l}\text { No } \\
\text { growth } \\
\text { observed }\end{array}$ & $\begin{array}{l}\text { Growth } \\
\text { observed }\end{array}$ & $\begin{array}{l}\text { Single } \\
\text { Candida } \\
\text { spp. } \\
\text { isolated }\end{array}$ & $\begin{array}{l}\text { Mixture of } \\
2 \text { Candida } \\
\text { spp. } \\
\text { isolated }\end{array}$ & $\begin{array}{l}\text { Total number } \\
\text { of Candida } \\
\text { spp .isolated }\end{array}$ \\
\hline $\begin{array}{l}\text { HIV } \\
\text { seropositive }\end{array}$ & 100 & 52 & 48 & 44 & $4(4 \times 2=8)$ & $44+8=52$ \\
\hline $\begin{array}{l}\text { HIV } \\
\text { seronegative }\end{array}$ & 50 & 26 & 24 & 20 & $4(4 \times 2=8)$ & $20+8=28$ \\
\hline
\end{tabular}

Table 2: Results of total number of Candida spp. isolated from HIV seropositive and seronegative patients.

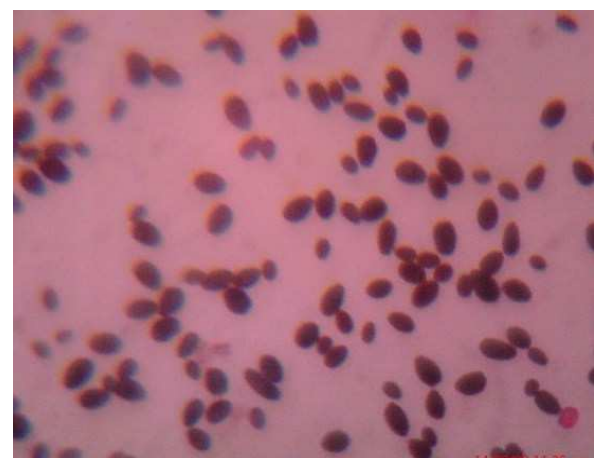

Figure- 1a

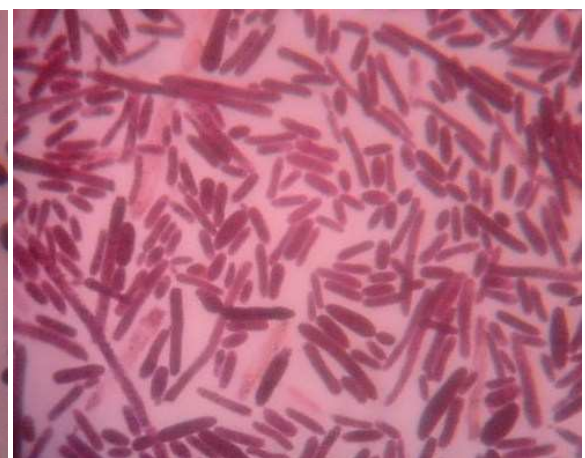

Figure-1b

Figure-1 : Gram staining from SDA (1a- Candida albicans, 1b- Candida krusei)

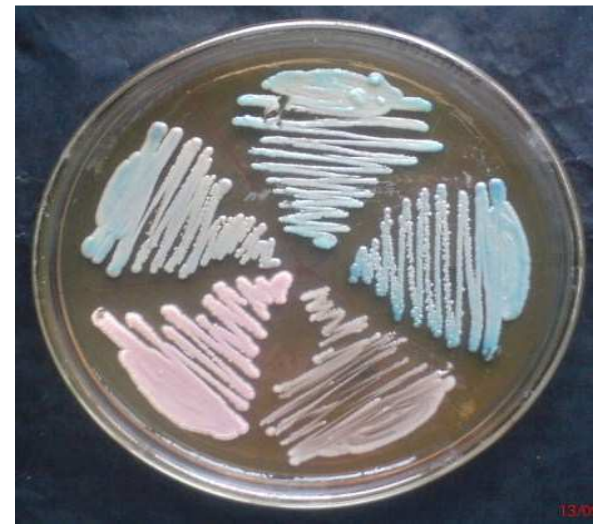

Figure-2 -different chromogenic reactions of various Candida species isolated. 


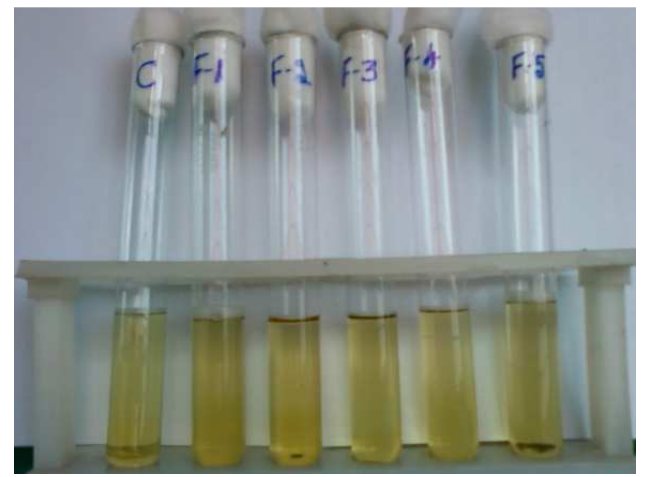

Figure-3 (Macrobroth dilution of antifungal (Fluconazole) susceptibility testing (break point method), showing- C- control without drug, F1-F5- various Candida isolates), F1 and F2 showing turbidity (resistant to Fluconazole), F3-F5- optically clear (susceptible to Fluconazole),

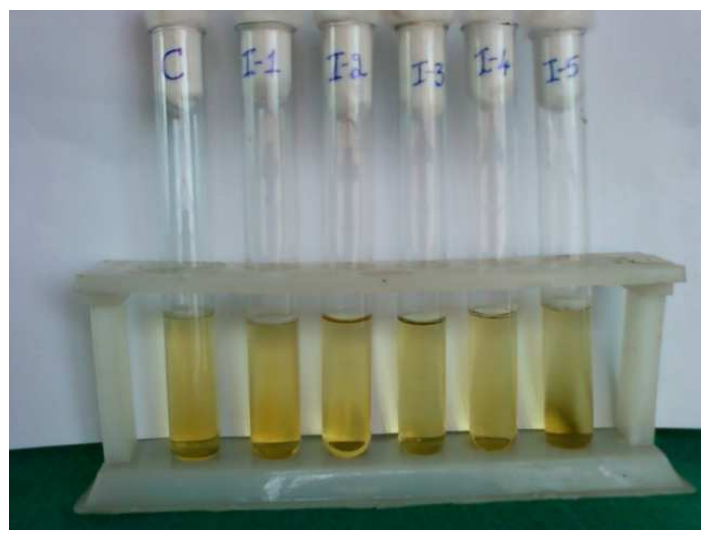

Figure-4 Macrobroth dilution of antifungal (Itraconazole) susceptibility testing (break point method) ,showing- C- control without drug, I1-I5- various Candida isolates, I1 showing turbidity (resistant to Itraconazole), I2-I5- optically clear (susceptible to Itraconazole)

\section{REFERENCE LISTS:}

1. Branchini ML, Pfaller MA, Rhine-Chalberg J, Frempong T, Isenberg HD. Genotypic variation and slime production among blood and catheter isolates of Candida parapsilosis. J Clin Microbiol 1994;32:452-456.

2. Raut SH, Varaiya A. Differentiationof Candida dubliniensis on Chrom agar and Pal,s agar. Indian Journal of Medical Microbiology;(2009)27(1):55-8.

3. Khan ZK, Gyanchandani A. Candidiasis-A Review. PINSA B64 No.1(1998): 1-34

4. Wabale V, Kagal A, Bharadwaj R. Characterization of Candida species from oral thrush in Human Immunodeficiency Virus (HIV) Seropositive and Seronegative Patients. Bombay Hospital Journal 2008;50(2):212-7.

5. Eraso E, Moragues MD, Villar-Vidal M, Sahand IH, Gonzalez-Gomez N, Ponton J, et al. Evaluation of the New Chromogenic Medium Candida ID2 for Isolation and Identification 
of Candida albicans and other Medically Important Candida species. Journal of Clinical Microbiology Sep2006;44(9):3340-5.

6. Murray MP, Zinchuk R Larone DH. CHOMagar Candida as the sole primary medium for isolation of yeasts and as a source medium for rapid-assimilation of trehalose test. J Clin Microbiol 2005; 43:1210-2.

7. Tortorano AM, Viviani MA, Barchiesi F, Arzeni D, Rigoni AL, Cogliati M, et al. Comparison of Three Methods for Testing Azole Susceptibilities of Candida albicans Strains Isolated Sequentially from Oral Cavities of AIDS Patients. Journal of Clinical Microbiology, 1998;36(6):1578-83.

8. Chunchanur SK, Nadgir SD, Halesh LH, Patil BS, Kausar Y, Chandrasekhar MR. Detection and antifungal susceptibility testing of oral Candida dubliniensis from human immunodeficiency virus-infected patients. Indian J Pathol Microbiol 2009;52(4):501-4.

9. Freicker-Hidalgol H, Orenga2 S, Lebeau1 B, Pelloux $1 \mathrm{H}$, Brenier-Pinchart $1 \mathrm{MP}$, Ambroise-Thomas1 P et al. Evaluation of Candida ID, a New Chromogenic Medium for Fungal Isolation and Preliminary Identification of Some Yeast Species. J Clin Microbiol.2001 April:39(4):1647-9.

10. Rippon JW. Candidiasis and the pathogenic yeasts. In: Martin Wonseiwicz editors. Medical Mycology. Philadelphia: W.B. Saunders; 1988. p. 531- 81

11. Khan ZU, Ahmad S, Mokaddas E, Al-Sweih N, Chandy R. Sunflower seed husk agar: A new medium for the differentiation of Candida dubliniensis from Candida albicans. Indian J Med Microbiol 2005;23:182-5.

12. Clinical and Laboratory Standards Institute. Reference Method for Broth Dilution Antifungal Susceptibility Testing of Yeasts;approved standard. Third Edition: Approved Standard M27-A3. Wayne, PA, USA: CLSI; 2008.

13. Rex JH, Pfaller MA, Walsh TJ, Chaturvedi V, Espinel-Ingroff A, Ghannoum MA, et al. Antifungal Susceptibility Testing: Practical Aspects and Current Challenges. Clin Microbiol Rev. 2001 October; 14(4): 643-58.

14. Rex JH, Walsh TJ, Sobel JD, et al. Practice Guidelines for the treatment of candidiasis. J Infect Dis. 2000; 30: 662-678.

15. Evci C, Ener B, Goral G, Akcaglar S. Comparative evaluation of the antifungal susceptibility of Candida isolates from blood specimens: results of a study in a tertiary care hospital in Bursa, Turkey. Turk J Med Sci 2010;40(1):141-9.

16. Latha R, Sasikala R, Muruganandam N, Venkatesh BR. Study on the shifting patterns of Non Candida albicans in lower respiratory tract infections and evaluation of the CHROMagar in Identification of the Candida Species. J. Microbiol. Biotech. Res 2011;1(3):113-9.

17. Willinger B, Manafi M. Evaluation of CHROMagar Candida for rapid screening of clinical specimens for Candida species.Mycoses1998;42:61-5.

18. Lopez-Dupla M, Sarz PM, Garcia VP, Ortega EV, Uriol PL, et al. Clinical, endoscopic immunologic and therapeutic aspects of oropharyngeal and esophageal candidiasis in HIV infected patients: A survey of 114 cases. Am J Gastroenterol 1992;87(12):1771-5.

19. Sullivan DJ, Westerneng TJ, Haynes KA, et al. Candida dubliniensis sp. now.:Phenotypic and molecular characterization of a novel species assosciated with oral candidiasisin HIV-infected individuals. Microbiol 1995;141:1507-21. 
20. Alves SH ,de Loreto ES, Linares CE, Silverira CP, Scheid LA, Pereira DI, et al. Comparison among Tomato juice agar with other three media for differentiation of C.dubliniensis from C.albicans. Rev Inst Med trop S Paulo 2006;48: 119-21.-

21. Odds FC, Bernaerts R. CHROMagar Candida, a new Differential Isolation Medium for presumptive Identification of Clinically Important Candida Species. Journal of Clinical Microbiology, Aug. 1994;32(8):1923-9.

22. Kirkpatrick WR, Revankar SG, Mcatee RK, Lopez-Ribot JL, Fothergill AW, McCarthy Di, et al. Detection of Candida dubliniensis in oropharyngeal samples from human immunodeficiency virus- infected patients in North America by primary chromagar Candida screening and susceptibility testing of isolates. J Clin Microbiol 1998;36:300712.

23. Albertson GD, Nimi M, Cannon RD, Jenkinson HF. Multiple Efflux Mechanisms Are Involved in Candida albicans-Fluconazole Resistance. Antimicrobial Agents and Chemotherapy, Dec.1996;40(12):2835-41.

24. Hamza OJ, Matee MI, Moshi MJ, Simon EN, Mugusi F, Mikx FH, et al. Species distribution and in vitro antifungal susceptibility of oral yeast isolates from Tanzanian HIV-infected patients with primary and recurrent oropharyngeal candidiasis. BMC Microbiol 2008;8: 135.

25. Pfaller MA. Nosocomial Candidiasis: Emerging Species, Reservoir, and Modes of Transmission. Clinical Infectious Diseases 1996;22(2):89-94.

26. Walmsley S, Kings, Mc Geer A, Ye Y, Richardson,S. Oropharyngeal Candidiasis in patients with Human Immunodeficiency virus: Correlation of clinical outcome with in vitro resistance, serum azole levels and immunosuppression. Clin Infect Dis 2001: 32 : 155461. 